\title{
Method of isolation and characterization of Girardia tigrina stem cells
}

\author{
K.A.R. LOPES ${ }^{1,2}$, N.M.R. de CAMPOS VELHO ${ }^{2}$ and C. PACHECO-SOARES ${ }^{2}$ \\ ${ }^{1}$ Laboratory Planarians, Nature Study Center, University of Vale do Paraíba; \\ ${ }^{2}$ Laboratory of Dynamics of Cellular Compartments, Institute of Research and Development, \\ University of Vale do Paraíba, São José dos Campos, SP 12244-000, Brazil
}

Received December 4, 2014; Accepted December 10, 2014

DOI: $10.3892 /$ br. 2014.408

\begin{abstract}
Tissue regeneration is widely studied due to its importance for understanding the biology of stem cells, aiming at their application in medicine for therapeutic and various other purposes. The establishment of experimental models is necessary, as certain invertebrates and vertebrates have different regeneration abilities depending on their taxon position on the evolutionary scale. Planarians are an efficacious in vivo model for stem cell biology, but the correlation between planarian cellular and molecular neoblast pluripotency mechanisms and those of mammalian stem cells is unknown. The present study had the following objectives: i) Establish Girardia tigrina cell culture, ii) determine the time required for complete cell disintegration and iii) obtain neoblasts by cell subdivision. Twenty-four specimens were deprived of food for seven days. After this time, disintegration was performed by incubation protected at three temperatures for $48 \mathrm{~h}$ in an antibiotic, antimycotic and trypsin solution, after which the suspension was homogenized and centrifuged. Histopaque ${ }^{\circledR} 1077$ was used for cell separation and interphases were collected and monitored by optical and fluorescence microscopy. Optical microscopy analysis informed the nucleus-to-cytoplasm ratio, cell morphology and cell size. Under fluorescence microscopy, interphase 1 (I1) was subdivided into two groups and neoblasts were marked for characterization; one group was stained with 4',6-diamidino-2-phenylindole and the other was immunolabeled with octamer-binding transcription factor 4 (OCT4) and isolated and observed after 10 days of cultivation. Neoblasts predominated in I1 with a small amount of other cell types. In conclusion, sample disintegration with a trypsin and antibiotic solution was effective at $18^{\circ} \mathrm{C}$ and Iscove's modified Dulbecco's medium supplemented with fetal bovine serum was adequate for the establishment of primary cell cultures after 48-h
\end{abstract}

Correspondence to: Dr Cristina Pacheco-Soares, Laboratory of Dynamics of Cellular Compartments, Institute of Research and Development, University of Vale do Paraíba, 2911 Av Shishima Hifumi, São José dos Campos, SP 12244-000, Brazil

E-mail: cpsoares@univap.br

Key words: stem cells, primary cell culture, Dugesiidae incubation and centrifugation. Antibody anti-OCT4 was used for the characterization of stem cells and was successfully labeled with concentrated neoblasts on interphase 1 .

\section{Introduction}

Regeneration is a complex event that occurs in several vertebrates and invertebrates (1). For regeneration to occur, one of the earliest signaling events following a lesion is the production of cells that are capable of rebuilding lost structures. The way that these events occur and the types of cells involved differ between animal groups (2).

The choice of planarians as a regeneration study model is based on their plasticity, regeneration ability, rapid response and adult stem cell reservoir (3). Cell and molecular characterization of planarian stem cells has been achieved with various tools, such as inhibition of the function of genes involved in regeneration via RNA interference, western blotting, in situ hybridization and cell isolation by flow cytometry (4-8). These tools are extremely important alternatives for understanding the mechanisms associated with the maintenance and role of stem cells in vivo (9).

Stem cells, also known as neoblasts, are located in the parenchyma and account for $\sim 20-30 \%$ of the whole cells of an individual and may present morphological subtypes (10-13). Neoblasts are characterized by i) the absence of morphological cytodifferentiation signs; ii) chromatoid body or large cytoplasmic ribonucleoprotein granules; iii) large nuclei with dispersed chromatin, or large nucleoli; iv) reduced cytoplasm containing numerous ribosomes; v) little endoplasmic reticulum; vi) perinuclear germ granules; and vii) X-ray sensitivity $(8,9,11,14)$.

The evolutionary conservation and expression of neoblasts in mammalian genes is associated with the maintenance and expression of pluripotency (15). Pluripotency transcription factors in mammalian stem cells are regulated by key genes, such as OCT4, SOX2 and NANOG. OCT4 is a member of the octamer-binding subgroup of the Pit-Oct-Unc transcription factor family $(16,17)$.

Due to a lack of information on the establishment of planarian primary neoblast cultures, particularly of Girardia tigrina, the present study proposed a methodology for cell culture establishment and characterization of neoblasts. 


\section{Materials and methods}

Animals. Specimens $(\mathrm{n}=24)$ of planarian Girardia tigrina were collected from the urban stretch of the Paraiba do Sul River in the municipality Jacarei (SP, Brazil). Specimen selection accounted for criteria, such as absence of lesions and perfect morphology. The selected animals were maintained in vials containing non-chlorinated water and were fasted for seven days until disintegration.

Disintegration and cultivation. The specimens were disintegrated as follows: 24 planarians were bathed in antibiotic and ultrapure water for $30 \mathrm{~min}$ each. The specimens were incubated in trypsin (Sigma-Aldrich, St. Louis, MO, USA) and antibiotic-antimycotic $\left(\right.$ Gibco $^{\circledast}$, Life Technologies, New York, NY, USA) (1:1; TA) in the dark for $48 \mathrm{~h}$. The partially disintegrated material was homogenized with a pipette and centrifuged (Excelsa Baby I; Fanem, Sao Paulo, SP, Brazil) at 2,360 $\mathrm{x}$ g for $5 \mathrm{~min}$. The cell sediment was resuspended in $1 \mathrm{ml}$ of Iscove's modified Dulbecco's medium (IMDM) supplemented with $10 \%$ fetal calf serum $\left(\mathrm{Gibco}^{\circledR}\right)$ and $1 \%(\mathrm{v} / \mathrm{v})$ antibiotic and antimycotic solution.

Cell viability. Cell viability was assessed with trypan blue (Sigma-Aldrich). Approximately 100 cells were counted in a Leica DM IL microscope (Leica Microsystems GmbH, Wetzlar, Germany), including stained and non-stained cells, to determine the proportion of membrane damage at different temperatures.

Cell fractionation. Following incubation in TA, the specimens were centrifuged and the segment was resuspended in $6 \mathrm{ml}$ of IMDM supplemented with $10 \%$ fetal calf serum and $1 \%$ antibiotic and antimycotic solution. For neoblast purification, $6 \mathrm{ml}$ of cells was suspended in $6 \mathrm{ml}$ of a solution of Histopaque ${ }^{\circledR} 1077$ (Sigma-Aldrich). The cell suspension was centrifuged (Fanem) at 3,248.93 x g for $45 \mathrm{~min}$. Following centrifugation, three interphases were visualized and cells were harvested from each one. This isolation procedure was performed in triplicate with five replications and cell culture was monitored for 10 weeks.

Analysis tools used to evaluate the culture for total and interphase cells. Disintegration and purification were complete after $48 \mathrm{~h}$ incubation in TA. The total cell culture samples and interphases were analyzed by optical and fluorescence microscopy. Optical microscopy showed the following cellular aspects: a) Nuclear cytoplasmic ratio; b) morphology; and c) cell size. For fluorescence microscopy, total cells and neoblast-enriched cell interphases were resuspended in $20 \mu \mathrm{l}$ sterile phosphate-buffered saline (PBS) and incubated with mouse anti-OCT4 (1:200) (AB-3209; Merck Millipore, Darmstadt, Germany) primary antibody for $60 \mathrm{~min}$ at room temperature. Subsequently, the cells were washed with PBS and incubated with fluorescein isothiocyanate-rat anti-mouse (04-6111; Invitrogen, Life Technologies, New York, NY, USA) secondary antibody at room temperature for $45-60 \mathrm{~min}$. Nuclei were detected by 4',6-diamidino-2-phenylindole (DAPI) (Sigma-Aldrich) staining. Images were captured using a Leica DM IL microscope fitted with a Leica DFC310 FX camera (Leica Microsystems $\mathrm{GmbH}$ ). After 10 days of cultivation,
$50 \mu \mathrm{l}$ of cell culture was collected in Eppendorf tubes and centrifuged for $5 \mathrm{~min}$ at 2,360 x g. Subsequently, the cells were resuspended and stained as previously described.

For scanning electron microscopy, $100 \mu \mathrm{l}$ of the control and irradiated groups were collected and centrifuged at $4,588.8 \mathrm{x} \mathrm{g}$ for $10 \mathrm{~min}$. Round coverslips were coated with a $0.1 \%$ solution of poly-L-lysine in water (molecular weight, 400,000; Sigma-Aldrich). The pellet was resuspended in PBS and the cells were placed on coverslips coated with poly-L-lysine and incubated for $20 \mathrm{~min}$ at room temperature. At the end of this period, the coverslips were washed to remove non-adhered cells, fixed with $0.25 \%$ glutaraldehyde and $2 \%$ paraformaldehyde (Sigma-Aldrich) in 0.1 M phosphate buffer and incubated for $10 \mathrm{~min}$. After fixation, the samples were washed with $0.1 \mathrm{M}$ phosphate buffer and dehydrated with acetone and hexamethyldisilazane for $10 \mathrm{~min}$. The coverslips were gold-palladium coated in a high vacuum evaporator on a rotating-tilting stage and observed in a Zeiss EVO MA 10 scanning electron microscope (Carl Zeiss Microscopy GmbH, Königsallee, Germany).

\section{Results}

Disintegration of the specimens. After an incubation period of $48 \mathrm{~h}$, the TA solution efficiently disintegrated the specimens and three regions with different levels of fragmentation were identified (Fig. 1). Following this period, the specimens were centrifuged to further total disintegration. Optical microscopy analysis revealed cell aggregates; some of which were isolated for characterization. The presence of mucus during cultivation following specimen disintegration had a double effect, as it contributed positively to cell adhesion to the bottom of the culture plate and hindered the characterization of cell types due to the formation of cell aggregates (Fig. 1B). Total cell decoupling occurred in four days.

Assessment of membrane integrity. Trypan blue was used due to the few mitochondria in the neoblasts. Membrane integrity was assessed through cell count. At the temperatures of 4, 18 and $37^{\circ} \mathrm{C}$, the cell membrane integrity was preserved with values of $63.81,51.92$ and $4.35 \%$, respectively.

Interphases following purification. Following complete purification, three interphases were obtained: Interphase 1 (I1), I2 and I3. Neoblasts predominated in I1 (Fig. 1C), with the presence of other cell types in smaller amounts. Fig. 1C shows that the neoblast nucleus was large and the cytoplasm was reduced, which was confirmed by fluorescent staining with DAPI (Fig. 2) and OCT-4 (Fig. 3A and B).

\section{Discussion}

The isolation of a pure neoblast cell fraction and its culture for a long period of time allowed the inclusion of Girardia tigrina among the model systems for the study of the stem cell signalization and genetic circuitry. However, there are limited studies on the establishment of cultures for the analysis of neoblast biology. Planarian Girardia tigrina, a species commonly found in Brazil, has been used as a model in our stem cells studies. 

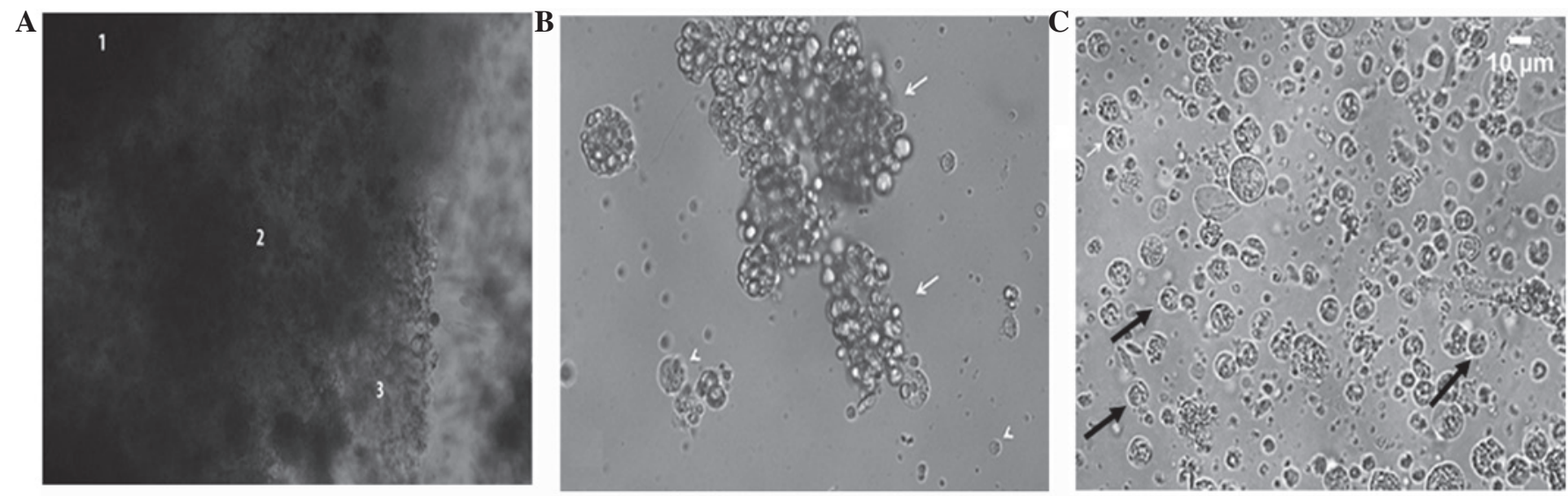

Figure 1. Disintegration and characterization of Girardia tigrina. (A) Cell culture. Specimens incubated in solution for $48 \mathrm{~h}$ showed different levels of disintegration, as indicated by numbers 1,2 and 3 . The disintegration of the external portion associated with mucus (1) and significant aggregation of cells in fragments 2 and 3 are observed. (B) The culture maintained for $48 \mathrm{~h}$ in Iscove's modified Dulbecco's medium (IMDM) supplemented with serum after disintegration in a trypsin and antibiotic-antimycotic (TA) solution showed free (arrow head) and aggregated cells (arrow). (C) Characterization of fragment 1 was constituted of neoblast-like cells (arrow) and other cell types. The fragment was cultivated in IMDM supplemented with serum fetal bovine.

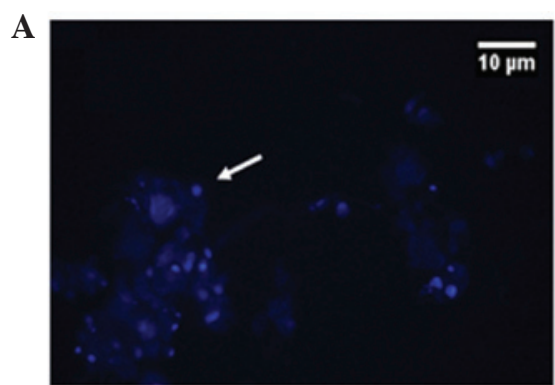

D

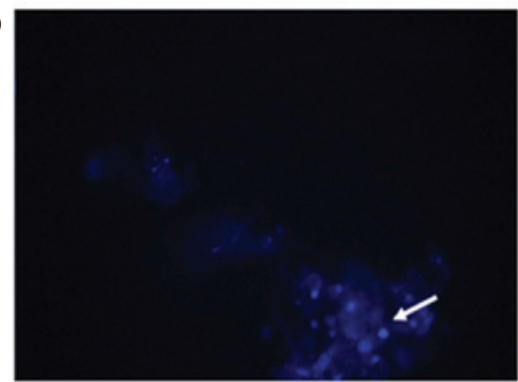

B

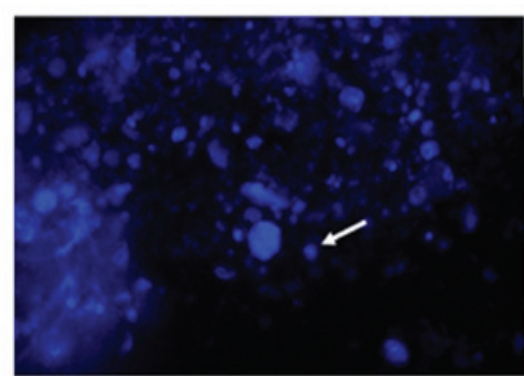

E

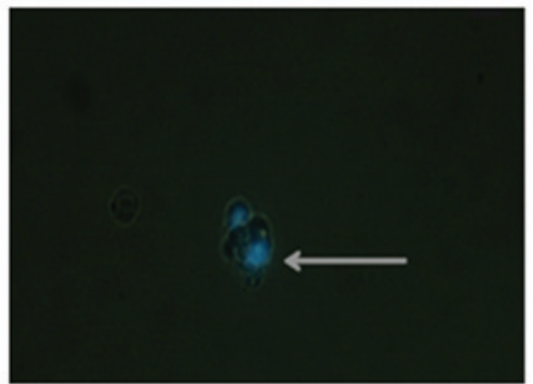

C

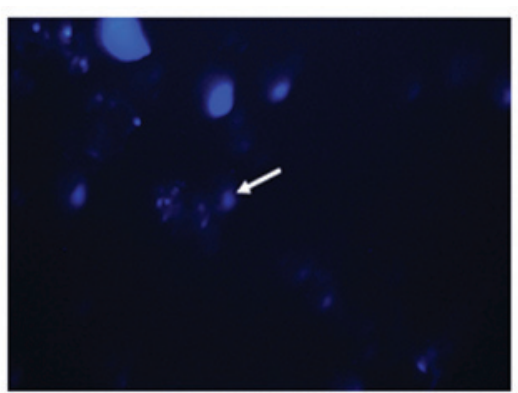

F

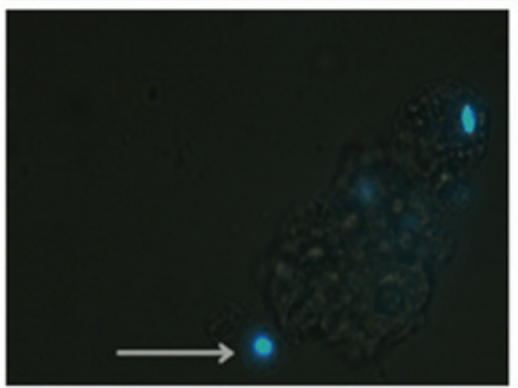

Figure 2. Neoblast characterization and culture. Neoblast characterization through 4',6-diamidino-2-phenylindole (DAPI) labeling. (A) Total culture neoblasts (arrow) with a high proportion of nucleus cytoplasm after $48 \mathrm{~h}$ of incubation. (B) Total culture after 10 days of incubation, several grouped cell types and isolated neoblasts (arrow). (C) Interphase 1 neoblast-like aggregates with large nuclei were effectively labeled with DAPI. (D) Neoblast-like aggregates with large nuclei after 10-day incubation. (E and F) Details of neoblasts isolated from interphase 1 (arrow).
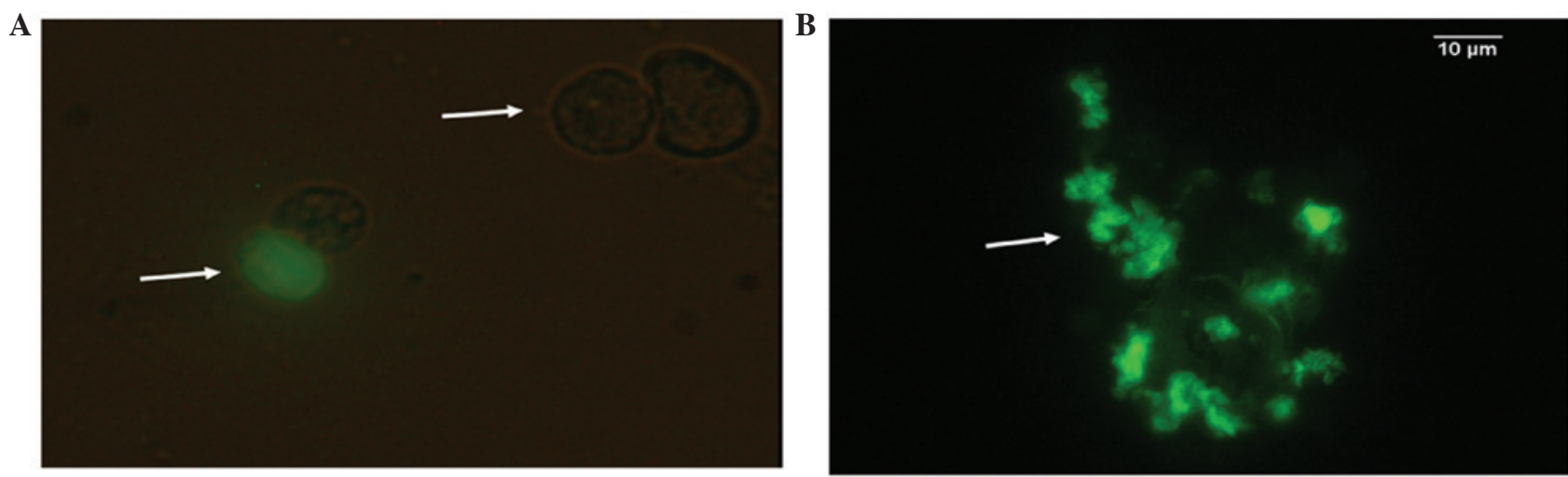

Figure 3. Neoblast characterization with octamer-binding transcription factor 4 (OCT-4). Neoblast characterization through anti-OCT-4 antibody immunolabeling. (A) Positive immunolabeling (neoblast) and negative immunolabeling cells for anti-OCT-4 (arrow). (B) Neoblast aggregate positive immunolabeling for anti-OCT-4 (arrow). 
The neoblast cultures were established from a planarian pool after fasting for 7-15 days, depending on the species used (4-6,18). Experiments with Dugesia tahitiensis and Dugesia polychroa used 24 -h baths $(7,8)$. In the present study, two 30-min antibiotic and sterile ultrapure water (1:1) baths were performed, in contrast to the aforementioned studies. Regarding the disintegration solutions and the cultivation medium, another relevant factor is the osmolarity relative to the studied specimen. Trypsin and pronase are digestive enzymes used to disperse cells and when specimens were incubated with trypsin $(0.25 \%)$ at $4^{\circ} \mathrm{C}$, testicular cell membrane damage and cell viability were reduced, demonstrating the effectiveness of this cell dissociation technique (9). The methodology adopted in the present study for the disintegration of Girardia tigrina involved incubation in a TA solution for $48 \mathrm{~h}$. Therefore, this is opposed to studies that used maceration, mechanical disintegration and enzymatic dissociation methods (8) and fragment disintegration in a Holtfreter's $5 / 8$ trypsin solution with subsequent gentle homogenization (5).

Trypsin may damage the cell membrane and thus affect the cell membrane integrity. In the present study, three incubation temperatures $\left(4,18\right.$ and $\left.37^{\circ} \mathrm{C}\right)$ were tested for disintegration in a TA solution and cell viability was assessed with trypan blue staining. At 4 and $18^{\circ} \mathrm{C}$, smaller damage rates occurred, respectively (9). Cell viability was only greatly compromised by $4.35 \%$ at $37^{\circ} \mathrm{C}$, confirming the thermal tolerance results obtained for Girardia tigrina. After $96 \mathrm{~h}$ at $33^{\circ} \mathrm{C},<50 \%$ of the specimens died (11).

Neoblast characterization through light and fluorescence microscopy showed rounded morphology and large nuclei when compared to the small cytoplasm, corroborating with several previous studies $(12,19)$. Besides morphological characterization, the neoblasts were labeled with antibody anti-OCT4 (Fig. 3), which is specific for mammalian stem cells and features homology to pluripotency factors and target genes between neoblast trascriptomes and proteomes (13). These results are consistent with those of others studies $(13,17)$, demonstrating that OCT4 is a good neoblast label, independent of the use of a planarian species as a model, as this gene evolutionarily conserved pluripotency.

In general, neoblasts comprise from $\sim 20$ to $30 \%$ of the cells that constitute the proliferative compartment known as blastema (7). The methods employed for disintegration and gradient centrifugation were efficient. Following purification, the proportion of neoblasts recovered in I1 was 15\%, in accordance with the studies mentioned above.

In conclusion, disintegration with a TA solution was efficient and $18^{\circ} \mathrm{C}$ is a suitable incubation temperature for Girardia tigrina specimens. IMDM supplemented with fetal bovine serum (10\%) was adequate for the establishment and maintenance of a planarian primary cell culture. Ultimately, antibody anti-OCT4 allowed the identification of neoblasts following purification in the Histopaque ${ }^{\circledR} 1077$ gradient and may be useful in regeneration and proliferation studies of stem cells applied to cell biology and tissue engineering.

\section{Acknowledgements}

The authors would like to thank the Foundation for Research Support of the State of Sao Paulo for grant no. 2009/15206-8 and National Council for Scientific and Technological Development for grant no. 480993/2010-6.

\section{References}

1. Isaeva VV, Akhmadieva AV, Aleksandriova IaN and Shukaliuk AI: Morphofunctional organization of reserve stem cells providing for asexual and sexual reproduction of invertebrates. Ontogenez 40: 83-96, 2009 (In Russian).

2. Wenemoser D and Reddien PW: Planarian regeneration involves distinct stem cell responses to wounds and tissue absence. Dev Biol 344: 979-991, 2010.

3. Salo E and Agata K: Planarian regeneration: a classic topic claiming new attention. Int J Dev Biol 56: 3-4, 2012.

4. Sato K, Shibata N, Orii H, et al: Identification and origin of the germline stem cells as revealed by the expression of nanos-related gene in planarians. Dev Growth Differ 48: 615-628, 2006.

5. Higuchi S, Hayashi T, Hori I, et al: Characterization and categorization of fluorescence activated cell sorted planarian stem cells by ultrastructural analysis. Dev Growth Differ 49: 571-581, 2007.

6. Tasaki J, Shibata N, Nishimura O, et al: ERK signaling controls blastema cell differentiation during planarian regeneration. Development 138: 2417-2427, 2011.

7. Behensky C, Schurmann W and Peter R: Quantitative analysis of turbellarian cell suspensions by fluorescent staining with acridine orange and video microscopy. Belg J Zool 131 (Suppl 1): 131-136, 2001.

8. Schurman W, Betz S and Peter R: Separation and subtyping of planarian neoblasts by density-gradient centrifugation and staining. Hydrobiologia 383: 117-124, 1998.

9. Terraciano PB, Paz AHR, Horn MM, et al: Isolation of rat testis cells from enzymatic digestion using trypsin at $4^{\circ} \mathrm{C}$. HCPA 24 : 163-164, 2004 (In Spanish).

10. Salo E and Baguna J: Regeneration and pattern formation in planarians. II. Local origin and role of cell movements in blastema formation. Development 107: 69-76, 1989.

11. de Campos Velho NMR: Planarians limnic (Tricladida: Dugesiidae) as models for regeneration experiments in microgravity and hypergravity environments (unpublished $\mathrm{PhD}$ thesis) Universidade do Vale do Rio dos Sinos, 2011

12. Pellettieri J and Sanchez Alvarado A: Cell turnover and adult tissue homeostasis: from humans to planarians. Annu Rev Genet 41: 83-105, 2007.

13. Rink JC: Stem cell systems and regeneration in planaria. Dev Genes Evol 223: 67-84, 2013.

14. Hayashi T, Asami M, Higuchi S, Shibata N and Agata K: Isolation of planarian X-ray-sensitive stem cells by fluorescence-activated cell sorting. Dev Growth Differ 48: 371-380, 2006.

15. Onal P, Grun D, Adamidi C, et al: Gene expression of pluripotency determinants is conserved between mammalian and planarian stem cells. EMBO J 31: 2755-2769, 2012.

16. Tantin D: Oct transcription factors in development and stem cells: insights and mechanisms. Development 140: 2857-2866, 2013.

17. Zhao FQ: Octamer-binding transcription factors: genomics and functions. Front Biosci (Landmark Ed) 18: 1051-1071, 2013.

18. Bender CE, Fitzgerald P, Tait SW, et al: Mitochondrial pathway of apoptosis is ancestral in metazoans. Proc Natl Acad Sci USA 109: 4904-4909, 2012.

19. Shibata N, Umesono Y, Orii H, Sakurai T, et al: Expression of vasa(vas)-related genes in germline cells and totipotent somatic stem cells of planarians. Dev Biol 206: 73-87, 1999. 